Japanese Journal of Applied Physics, 33 (11) (1994) 6299-6300

\title{
ESR of Photodarkened CdS-Doped and Undoped Glasses
}

Tadaki Miyoshi, Ken-ichi Towata and Naoto Matsuo

Department of Electrical and Electronic Engineering, Yamaguchi University, Tokiwadai, U be, Yamaguchi 755

(Received June 17, 1994; accepted August 20, 1994)

Electron spin resonance (ESR) and luminescence were measured in CdS-doped glass and undoped glass, which does not contain $\mathrm{CdS}$ microcrystals. Photoinduced centers responsible for the photodarkening effects were detected by ESR in the CdS-doped glass. This ESR signal is different from that of the host glass. This result indicates that the centers responsible for the photodarkening are not associated with the centers in the glass.

KEYWORDS: phot odar keni ng, semi conduct or, quant um dot, ESR, I um nescence 
In recent years semiconductor-doped glasses have been studied extensively as nonlinear-optical materials. The optical nonlinearity and the response time are decreased by light irradiation. These photoinduced processes are called photodarkening effects. The photodarkening effects are considered to be due to traps in the host glass. ${ }^{1,2)}$ Here, we report the measurements of ESR and luminescence of CdS-doped and undoped glasses to investigate the validity of the model. We have already observed the photoinduced centers in CdSSe-doped glass after light irradiation using ESR. ${ }^{3)}$

The samples investigated were a CdS-doped commercial glass filter (Toshiba Y-45) and host glass (Y-0) for the filter with thickness of $2.5 \mathrm{~mm}$. While Y-45 contains CdS microcrystals with diameter of about $10 \mathrm{~nm}$, Y-0 does not contain CdS microcrystals. The CdS-doped glass was exposed to pulsed light from an $\mathrm{N}_{2}$ laser (NDC JS-1000L; wavelength $=337.1 \mathrm{~nm}$, pulse duration $=5 \mathrm{~ns}$, peak intensity $=4 \mathrm{MW} / \mathrm{cm}^{2}$, repetition rate $=4 \mathrm{~Hz}$ ) at $300 \mathrm{~K}$ for $20 \mathrm{~min}$. Transmittance of the glass is less than $10^{-7}$ at a wavelength of $337.1 \mathrm{~nm}$. Thus the penetration depth of $\mathrm{N}_{2}$ laser light is less than 0.16 $\mathrm{mm}$. The host glass was exposed to pulsed light from a KrF laser (wavelength $=248 \mathrm{~nm}$, pulse durations $=15 \mathrm{~ns}$, peak intensity $=5 \mathrm{MW} / \mathrm{cm}^{2}$, repetition rate $=10 \mathrm{~Hz}$ ) at $300 \mathrm{~K}$ for $10 \mathrm{~min}$. $\mathrm{KrF}$ laser light is absorbed at the surface region of the glass.

The ESR spectra were measured at $77 \mathrm{~K}$ using an X-band spectrometer (JES FE-1X). The first derivative spectra were obtained by a $100 \mathrm{kHz}$ field modulation. Transient characteristics of luminescence were measured using the following apparatus at $77 \mathrm{~K}$ and $300 \mathrm{~K}$. The excitation source was an $\mathrm{N}_{2}$ laser (Laser Photonics LN120; wavelength $=337.1 \mathrm{~nm}$, pulse duration $=300 \mathrm{ps}$, repetition rate $=7 \mathrm{~Hz}$ ). The laser beam was set at an angle of about $30^{\circ}$ off the normal incidence to the surface of the sample and was focused on an area about $1 \mathrm{~mm}^{2}$ by a quartz lens (focal length $\mathrm{f}=150 \mathrm{~mm}$ ). The peak intensity of the laser light on the sample was about $50 \mathrm{~kW} / \mathrm{cm}^{2}$ (photodarkening effects were not observed at this intensity). Luminescence was collected normal to the sample surface, focused on the end of an optical fiber by a quartz lens ( $f=50.8 \mathrm{~mm}$ ), and then led to the entrance slit of a $27 \mathrm{~cm}$ monochromator (Jarrell-Ash Monospec 27). Time-resolved luminescence spectra were measured using an optical multi-channel analyzer with gate (Princeton Instruments D/SIDA-700). The minimum gate time is 5 ns. 
Figure 1 (a) shows the ESR spectra of the unirradiated and light-irradiated CdS-doped glasses at $77 \mathrm{~K}$. The ESR signals appear by light irradiation. The g-values of the signals are determined using a MgO:Mn marker. The ESR signals except for A and B disappear after annealing at $200{ }^{\circ} \mathrm{C}$ for $15 \mathrm{~min}$, and signals $\mathrm{A}$ and $\mathrm{B}$ disappear after annealing at $400{ }^{\circ} \mathrm{C}$ for $2 \mathrm{~h}$.

A luminescence peak is observed at $440 \mathrm{~nm}$, which is the shallow-trap band. ${ }^{4)}$ Figure 2 shows luminescence intensities at $440 \mathrm{~nm}$ as a function of time at $300 \mathrm{~K}$. The decay rate of the $440 \mathrm{~nm}$ band increases after light irradiation. Although the decay rate does not recover its initial value after annealing at $200{ }^{\circ} \mathrm{C}$ for $15 \mathrm{~min}$, it recovers after annealing at $400{ }^{\circ} \mathrm{C}$ for $2 \mathrm{~h}$. This correlation between the intensity of the ESR signals A and $\mathrm{B}$ and the response time in luminescence suggests that the centers responsible for the ESR signals A and B are associated with photodarkening effects.

Figure 1 (b) shows the ESR spectrum of light-irradiated host glass at $77 \mathrm{~K}$. The ESR signal appears by $\mathrm{KrF}$ laser light irradiation and does not appear by $\mathrm{N}_{2}$ laser light irradiation. The signal is attributable to photoinduced centers in the host glass. This signal is probably due to trapped hole centers, which have been observed in X-ray-irradiated alkali silicate glasses. ${ }^{5)}$ The g-value of the signal is different from those in the CdS-doped glass. Moreover, the diminishing temperature of the centers is about $200{ }^{\circ} \mathrm{C},{ }^{5)}$ which is lower than that for signals A and B. These results indicate that signals $\mathrm{A}$ and $\mathrm{B}$ in the CdS-doped glass are not associated with the trap centers in the glass matrix. Signals A and B may be due to the centers in the CdS microcrystals or the interface region between the $\mathrm{CdS}$ microcrystals and the glass matrix. We measured ESR spectra of CdS fine particles with diameter of about 100-1000 nm. However, we did not observe the effect of light irradiation on the ESR spectrum. Thus signals A and B are probably due to the centers in the interface region between CdS microcrystals and glass matrix.

Decrease in transmittance is observed in the host lass irradiated by $\mathrm{KrF}$ laser light. On the other hand, change in transmittance is not observed in the CdS-doped glass irradiated by $\mathrm{N}_{2}$ laser light. This transmission change may be due to trapped electrons (or holes) in the glass matrix. Thus it is likely that a large number of electrons (holes) are trapped in the host glass and a small number of electrons (holes) are trapped in the 
glass matrix of the CdS-doped glass. We consider that the traps in the glass matrix change transmittance and the photoinduced centers in the semiconductor-glass interface change response time of luminescence.

Recently, Yanagawa and Nakano ${ }^{6)}$ reported the ESR spectrum of photodarkened CdSSe-doped glass (Hoya O-54). They observed three ESR signals with $\mathrm{g}=1.990$, 1.999 and 2.010. They assigned the signals at $\mathrm{g}=1.990$ and 2.010 to electron and hole traps in the interface region, respectively. These g-values except for $\mathrm{g}=1.990$ are different from those in the present result of CdS-doped glass. The discrepancies are probably due to the difference in compositions of glass matrices and semiconductor microcrystals. A comparative study of Toshiba and Hoya glasses is in progress to elucidate the origin of the discrepancies.

In summary, ESR and luminescence of CdS-doped and undoped glasses are reported. Centers responsible for the photodarkening effects are different from those in the host glass. This result indicates that the photodarkening effects are probably due to the photoinduced centers at the glass-semiconductor interface.

The authors are grateful to Dr. A. Kai for her cooperation in the ESR experiment and for valuable comments. The permission of Dr. Y. Aoyagi and Dr. S. Komuro to use their KrF laser in RIKEN (The Institute of Physical and Chemical Research) is gratefully acknowledged. 
R eferences

1) V. Ya. Grabovskis, Ya. Ya. Dzenis, A. I. Ekimov, I. A. Kudryavtsev, M. N. Tolstoi and U. T. Rogulis: Sov. Phys. Solid State 31 (1989) 149.

2) J. Malhotra, D. J. Hagan and B. G. Potter: J. Opt. Soc. Am. B 8 (1991) 1531.

3) T. Miyoshi and T. Miki: Jpn. J. Appl. Phys. 31 (1992) 1461.

4) K. Misawa, H. Yao, T. Hayashi and T. Kobayashi: Chem. Phys. Lett. 183 (1991) 113.

5) J. W. H. Schreurs: J. Chem. Phys. 47 (1967) 818.

6) T. Yanagawa and H. Nakano: Mater. Res. Soc. Symp. Proc. 318 (1994) 495. 
Figure captions

Fig. 1. ESR spectra of glasses at $77 \mathrm{~K}$ : (a) CdS-doped glass (Y-45) before and after irradiation, (b) undoped glass (Y-0) after irradiation.

Fig. 2. Luminescence intensities at $440 \mathrm{~nm}$ of CdS-doped glass as a function of time at $300 \mathrm{~K}$ : before irradiation (open circles), after irradiation (solid circles) and after annealing at $400{ }^{\circ} \mathrm{C}$ for $20 \mathrm{~min}$ (squares). Solid curves were drawn through data points as guide to the eyes. Intensities at delay time $t_{d}=0$ are normalized. 
Fig. 1

a) $Y-45$

before irrad.

$\mathbf{N}_{2}$ laser

A B

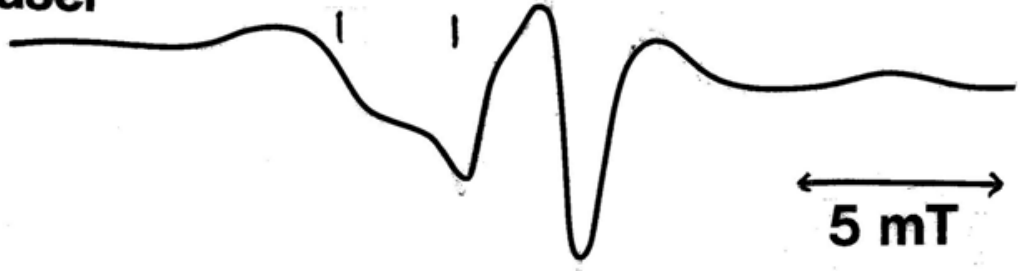

b) $Y-O$

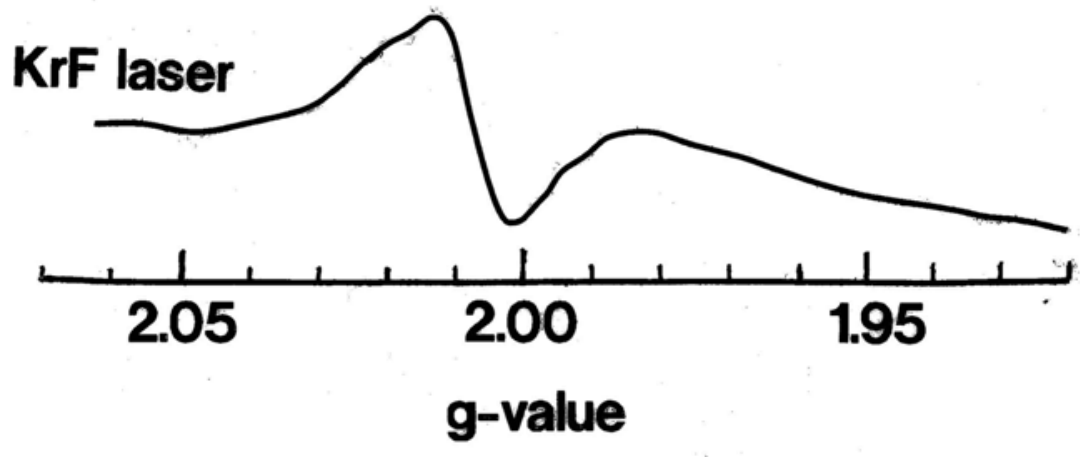

Fig. 2

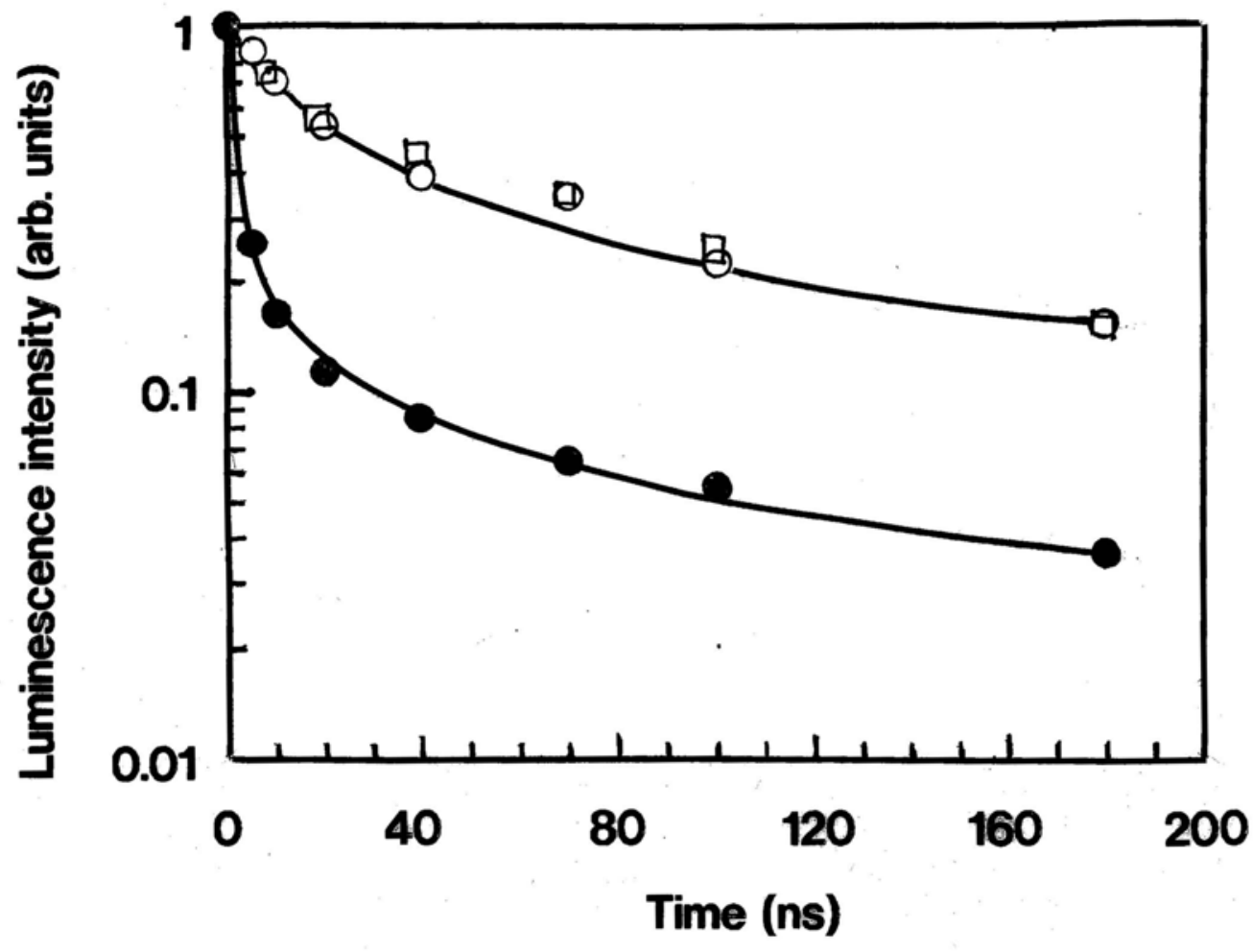

